\title{
Parce que RIPES ne serait pas ce qu'elle est sans elle... Merci Brigitte!
}

Christelle Lison

\section{(2) OpenEdition}

\section{Journals}

Édition électronique

URL : http://journals.openedition.org/ripes/1926

DOI : 10.4000/ripes.1926

ISSN : 2076-8427

Éditeur

Association internationale de pédagogie universitaire

Référence électronique

Christelle Lison, « Parce que RIPES ne serait pas ce qu'elle est sans elle... Merci Brigitte! », Revue internationale de pédagogie de l'enseignement supérieur [En ligne], 35(1) | 2019, mis en ligne le 17 mai 2019, consulté le 25 septembre 2020. URL : http://journals.openedition.org/ripes/1926 ; DOI : https:// doi.org/10.4000/ripes. 1926

Ce document a été généré automatiquement le 25 septembre 2020.

Article L.111-1 du Code de la propriété intellectuelle. 


\title{
Parce que RIPES ne serait pas ce qu'elle est sans elle... Merci Brigitte!
}

\author{
Christelle Lison
}

1 Vous ne le savez peut-être pas, mais le modèle de fonctionnement de RIPES, qui est la revue officielle de l'Association Internationale de Pédagogie Universitaire (AIPU) est maintenant une revue en ligne depuis dix ans. Depuis toutes ces années, des personnes travaillent bénévolement, ou en étant soutenu par des institutions d'enseignement supérieur, pour faire que RIPES soit une revue de qualité. Parmi les chevilles ouvrières de la revue, il y a une personne discrète, dont vous n'avez probablement jamais entendu parler... Et pourtant, chers auteurs et chers lecteurs, elle est une personneclé... Son nom : Brigitte Goudrange.

2 Avec son bagage en biologie et sa passion pour l'enseignement et l'apprentissage, Brigitte Gourdange a rejoint l'équipe du LabSET de l'Université de Liège en mars 2009 à titre de conseillère pédagogique. Depuis cette date, elle assurait la coordination informatique de RIPES. Vous avez bien lu, oui, après plus de dix ans au cœur même de la revue, Brigitte nous quitte pour d'autres aventures. Mais pourra-t-elle réellement nous quitter? Probablement pas! Elle nous lira avec plaisir, nous l'espérons. Une chose est sûre, Brigitte nous manquera. Ses compétences, son efficacité, sa gentillesse... Personnellement, je n'ai jamais connu RIPES sans Brigitte... J'appréhende un peu ce changement, mais je le comprends tout à fait. Je n'ai qu'un mot, à la fois court et profond, merci Brigitte de m'avoir accompagnée depuis toutes ces années et ma prédécesseure avant moi. À cette occasion, je ne peux passer sous silence l'implication du LabSET de l'Université de Liège pour RIPES. Merci de nous avoir permis de bénéficier de la présence de Brigitte à nos côtés. La revue ne serait certainement pas ce qu'elle est, sans elle. Et je ne serais certainement pas la rédactrice en chef que je suis...

Pour ce premier numéro de l'année 2019, une année qui s'annonce forte en émotions, vous aurez l'occasion de découvrir six articles et deux notes de lecture. Vous nous permettrez, pour cette occasion, de commencer ce numéro par un article de collègues de l'Université de Liège (Belgique), Gilles Fossion et Ariane Baye. Celui-ci porte sur les exemples dans le discours pédagogique, et les impacts cognitifs et motivationnels de 
ceux-ci sur les étudiants. Pour vérifier leurs hypothèses, les auteurs ont mis en place un protocole expérimental auprès de 229 étudiants universitaires. Ils en arrivent à la conclusion que les exemples, et certains en particulier, sont efficaces pour favoriser la compréhension et la motivation des étudiants.

Le deuxième article en est également un de collègues belges, de différentes institutions, tous membre du groupe AdAPTE. Dans leur texte, Mireille Houart, Sylviane Bachy, Sylvie Dony, Isabelle Lambert, Chantal Poncin et Pauline Slosse s'intéressent à la manière dont les étudiants gèrent les conditions dans lesquelles ils se placent pour réaliser les activités d'apprentissage en autonomie et plus particulièrement la réalisation de synthèses. À partir des liens entre volition, cognition et motivation, qu'elles mettent en lumière, à travers l'analyse des questionnaires collectés auprès de 2200 étudiants de première année répartis dans 16 établissements d'enseignement supérieur, les auteures en arrivent à dégager des pistes pour rendre les étudiants plus actifs, plus attentifs et plus persévérants.

5 Le troisième article, de Nicole Monney et Nadia Cody, toutes deux de l'Université du Québec à Chicoutimi, présente les résultats d'une recherche collaborative qui met en scène une équipe de multiacteurs (enseignants associés, superviseurs universitaires et chercheures) pour structurer un eportfolio et procéder à sa mise à l'essai dans le cadre d'une formation de premier cycle en éducation préscolaire et enseignement primaire. Cette recherche soulève notamment des questions quant à la perception du rôle de l'enseignant associé au cœur du processus et à l'accompagnement offert aux stagiaires pour développer leur posture réflexive; questions qui mériteraient d'être discutées entre enseignants associés, superviseurs universitaires et chercheurs.

6 Le quatrième article, de collègues de l'Université Laval, Josée-Anne Gouin et Christine Hamel, s'intitule "Convergences et divergences dans la phase de la cosituation d'une recherche entre des formateurs de stagiaires en enseignement secondaire ». Proposant elle aussi une démarche de recherche collaborative, les auteures ont mis en place une communauté de pratique composée de sept formateurs de stagiaires. En observant la négociation de sens en cours d'action entre les personnes, Gouin et Hamel dégagent des zones de convergence ainsi que des zones de divergence entre les acteurs. Elles en arrivent à constater le défi que représente pour les responsables de stage le fait d'amener les formateurs de stagiaires, les enseignants associés et les superviseurs universitaires à travailler en collaboration.

7 Le cinquième article porte sur les retombées de l'autoévaluation accompagnée et contractualisée sur le développement professionnel des futurs enseignants et formateurs d'adultes. Dans ce texte, Bruno Perrault (Université d'Artois) et Thérèse Levené (Université de Lille) partent du constat que la rédaction du mémoire professionnel par les enseignants et les formateurs d'adultes en formation initiale ne garantit pas à elle seule le développement d'une posture réflexive régulatrice des pratiques. Ils font en fait l'hypothèse du développement de la pensée réflexive par l'autoévaluation des pratiques. Les résultats de la recherche qu'ils ont menées par le biais d'entretiens les amènent à mettre en lumière les conditions de réussite de la démarche d'autoévaluation.

8 Le sixième article est proposé par Marion M. Bisiaux, Laetitia Fontaine et Nicole Rege Intitulé "Enseigner la sortie de cadre pour changer les habitudes", il s'agissait d'intégrer, dans l'ingénierie pédagogique de l'atelier matières à construire - amàco, les principes d'une philosophie de la matière qui invite à faire corps avec celle-ci. Après 
trois années d'expérimentation pédagogique, une démarche d'investigation, basée sur la théorie ancrée, a été entreprise pour observer, d'une part, les effets sur l'expérience formative des étudiants et le développement de leurs compétences professionnelles et, d'autre part, les modifications des pratiques d'enseignement des enseignants impliqués dans le projet. L'analyse montre que les changements les plus apparents concernent les enseignants qui transforment radicalement leur enseignement au-delà de l'expérience. Les effets sur le développement professionnel des étudiants sont quant à eux plus difficiles à saisir bien que prometteurs.

9 Finalement, pour ce premier numéro de l'année 2019, deux recensions de lecture vous sont proposées. Tour d'abord, Charlotte Pourcelot, de l'Université Paris-Est (France), discute de l'ouvrage de Patrick Fauconnier, intitulé « Universités : innover ou sombrer. Ensuite, Constance Denis, de l'Université de Sherbrooke (Canada), propose une recension du dernier livre de Christian Bégin « Encadrer aux cycles supérieurs: Étapes, problèmes et interventions". 